Peer Reviewed Paper openaccess Special Issue on Spectral Imaging in Synchrotron Light Facilities

\title{
Performance comparison of aperture-less and confocal infrared microscopes
}

\author{
C. Sandt, ${ }^{a *}$ Z. Dionnet, ${ }^{b}$ M. Toplak, ${ }^{c}$ E. Fernandez, ${ }^{d}$ R. Brunetto ${ }^{e}$ and F. Borondics ${ }^{\dagger}$ \\ aSMIS beamline, Synchrotron SOLEIL, L'orme des merisiers, 91192 Gif sur Yvette, France. E-mail: sandt@synchrotron-soleil.fr, \\ (1) https://orcid.org/0000-0002-6432-2004 \\ bIAS, Université Paris-sud, Orsay, France. 는 https://orcid.org/0000-0002-3679-6333 \\ 'SMIS beamline, Synchrotron SOLEIL, L'orme des merisiers, 91192 Gif sur Yvette, France. 은ttps://orcid.org/0000-0003-4413-1603 \\ ${ }^{d}$ Yves Rocher, Direction Innovation \& Développement, 7 Chemin de Bretagne, Issy les Moulineaux, France \\ eSMIS beamline, Synchrotron SOLEIL, L'orme des merisiers, 91192 Gif sur Yvette, France \\ fSMIS beamline, Synchrotron SOLEIL, L'orme des merisiers, 91192 Gif sur Yvette, France. 은 https://orcid.org/0000-0001-9975-4301
}

\begin{abstract}
We compared hyperspectral infrared raster maps and images for contrast, definition and resolution of the same samples recorded with a confocal microscope coupled with a synchrotron radiation source vs a Focal Plane Array (FPA) detector equipped microscope. Biological samples (hair and skin sections) and astrophysics samples (meteoritic grains) were used. The samples presented are a few microns in size, such as embedded particles, a single unique cell or thin layer. Our results show that the actual spatial resolution and contrast of FPA images were lower than spectral maps from the confocal microscope. The FPA microscope also produced measurements that lacked accuracy: size of sample features and peak intensity were inaccurately estimated. More surprisingly, the intensity of absorption peaks in the FPA images was lower than the intensity measured from the same sample with a confocal microscope. Our measurements underlined the complementarity of FPA and confocal microscopes. FPA can be used to quickly measure the overall composition of a sample and detect the distribution of its components, but may fail measuring the exact chemical composition of the small features and may not detect weak spectral differences between adjacent positions. The averaging effect of aperture-less systems not only affects image resolution but also lowers their spectral accuracy. Confocal microscopes are inherently slower but give a more accurate measurement of the local composition at the diffraction limit.
\end{abstract}

Keywords: infrared microspectroscopy, imaging, FPA, confocal, synchrotron radiation, spatial resolution, diffraction limit

\section{Introduction}

Spatial resolution is a key parameter in any spectral imaging experiment since it defines the smallest feature size that can be measured accurately without contamination from the surrounding medium or neighbouring particles. The best spatial resolution achieved by any microscope in the far-field is foremost limited by diffraction and the performances of the optical system, and is summarised by the famous Abbe equation:

$$
R=\frac{1.22 \lambda}{2 N A}
$$

Correspondence

C. Sandt (sandt@synchrotron-soleil.fr)

Received: 15 February 2019

Revised: 8 March 2019

Accepted: 11 March 2019

Publication: 1 April 2019

doi: $10.1255 /$ jsi.2019.a8

ISSN: 2040-4565

\section{Citation}

C. Sandt, Z. Dionnet, M. Toplak, E. Fernandez, R. Brunetto and F. Borondics, "Performance comparison of aperture-less and confocal infrared microscopes", J. Spectral Imaging 8, a8 (2019). https://doi.org/10.1255/jsi.2019.a8

(c) 2019 The Authors

This licence permits you to use, share, copy and redistribute the paper in any medium or any format provided that a full citation to the original paper in this journal is given. 
where $\lambda$ is the wavelength and NA the numerical aperture of the optical system.

Infrared microscopes use Schwarzschild or Cassegrain all-reflective objectives, which block the central part of the infrared beam giving a better theoretical spatial resolution (for example, $R=1.0 \lambda / 2$ NA using a $32 \times$ Schwarzschild objective with a linear obscuration ratio of 0.4 as used in this study) at the expanse of the signal throughput. ${ }^{1}$ In addition, the confocal configuration further improves the lateral resolution to approximately $\lambda / 2$ as shown in the study of G.L. Carr. ${ }^{1}$

Infrared microspectroscopy is classically performed in confocal microscope configuration and using a single-point mercury cadmium telluride (MCT) detector. Rastered hyperspectral maps are measured in successive acquisitions, i.e. one spectrum at a time. On one hand, the confocal configuration reduces signal throughput, restricts the field of view and imposes the measurement of a single point at a time. Yet, it is crucial to eliminate diffraction and to achieve lateral resolution at the diffraction limit as well as to improve the axial resolution. ${ }^{1-3}$ On the other hand, infrared imaging microscopes equipped with Focal Plane Array (FPA) detectors can acquire hyperspectral images containing thousands of spectra in a matter of minutes. They give high-definition spectral images (definition being the number of pixels in the image) that appear to have greater spatial resolution than the spectral maps generated by confocal systems. ${ }^{4-6}$ This is counter-intuitive, since FPA microscopes do not have a confocal aperture and thus each pixel in the detector may be affected by the diffraction created by optics and fibrillary and particulate materials (cells, organelles, grains) in the sample. In addition, pixels in the detectors are physical objects with edges that can create some diffraction affecting the neighbouring pixels. A third, intermediate, configuration exists in the form of linear array detectors that can acquire several spectra simultaneous (8-32 in most common commercial configurations), but still require multiple sequential measurements to be performed in order to create a map of the whole sample. Staggered array detectors can be designed with pixels separated by a few microns to avoid recording diffraction rings (as in the Thermo Scientific iN10MX microscope and PerkinElmer Spotlight), but this could not totally prevent diffraction from the rest of the optical system to reach the detector.

The spatial resolution of FPA detectors depends on the detector configuration (physical pixel size and spacing), microscope optics (objective numerical aperture and obscuration ratio, optical path) but also from the diffraction from the sample. Thus, it has most often been established by measurements with the step-edge method, ${ }^{1,7-11}$ by using a model 1951 USAF resolution target consisting of an infrared reflective photoresist layered on a glass substrate. Spatial resolutions below the diffraction limits were reported, such as $2.4 \mu \mathrm{m}$ at $3794 \mathrm{~cm}^{-1}$ (2.5 $\mathrm{mm}$ wavelength) in Lasch et al. ${ }^{8}$ with a Bruker Hyperion microscope and $2.19 \mu \mathrm{m}$ at $3794 \mathrm{~cm}^{-1}$ in Nasse et al. ${ }^{9}$ These values were well above the best theoretical value achievable by such systems, as noticed by Lasch and coworkers. However, since the USAF model 1951 resolution target is made of fully reflective bars surrounded by fully absorbing glass, it is hardly comparable to most of the relevant samples composed of chemically and spectrally very similar constituents and resolution measurements using this target may yield overestimated values. ${ }^{12,13}$

FPA images may exhibit an extremely high definition, for example the FPA detector used in this study has a definition of 16,384. High-definition images may easily be confused with highly spatially resolved images, since they display a large number of pixels in a given area. However, this area is generally larger than the diffraction limit of the system and is not actually resolvable in smaller details. This situation is called oversampling and high-definition FPA images may be highly oversampled. For example, the system used in this study can give a $0.65 \times 0.65 \mu \mathrm{m}^{2}$ projected pixel size by using a $25 \times$ NA 0.81 objective in high-magnification mode. Such an objective would give an optimal spatial resolution of $12.3 \mu \mathrm{m}$ at $10 \mu \mathrm{m}$ wavelength. This means that one Airy pattern would be imaged by 38 pixels and the first Airy disk by 281 pixels. While some level of oversampling may be necessary to achieve optimal sampling (Nyquist criterion) and better contrast (Stelzer criterion $)^{14}$ in images, it is difficult to estimate if there would be meaningful spectral variations through such a pattern, especially for the wide spectral domain covered by Fourier transform infrared (FT-IR) microspectroscopy. Here, we report measurements performed with both an aperture-less FPA system and a confocal aperture microscope coupled to a synchrotron source on a selection of representative samples. We evaluate the spectral accuracy of the spectra from micron-sized features as measured by the aperture-less system at different magnifications and by the confocal infrared microscope. 
We define spectral accuracy as the closeness of the infrared spectrum of a given position to the real spectrum at the same position and use the confocal microscope as our "gold standard". Samples were selected to be representative of different architectures (multilayered and/or particulate) with feature sizes comparable to the diffraction limit in the $2.5-15 \mu \mathrm{m}$ region of the mid-infrared spectral range. Biological samples naturally exhibit features at the micron scale and generally present weak chemical contrasts as different compartments of the sample are composed of slightly different mixtures of the same types of biomolecules. We selected hair samples to represent particulate architecture, and skin samples for layered architecture. We also used a fragment from the famous Paris meteorite containing various inclusions with different chemical compositions (calcium carbonate, calcium sulfate, serpentine...). ${ }^{15}$

We report significant differences between spectra of small inclusions and thin layers when comparing FPA images and the confocal maps from the same sample. Even at the shortest wavelength, those less affected by diffraction, FPA spectra did not always represent the chemical composition of a finely microstructured sample accurately. In addition, we observed that the overall peak intensity of the hair and skin sections was lower when the samples were measured with FPA than with the confocal system.

\section{Experimental Samples}

Hair preparation

The human hair samples consisted of natural hair fibres of European origin. A compact wet hair bundle was assembled in a plastic capillary, then frozen. The ice-embedded hair bundle was mounted in the cryostat chamber and sectioned. Three-micron-thick cross-sections were collected on $1 \mathrm{~mm}$ thick ZnS slides and stored at ambient temperature prior to IR analysis. The samples were kindly provided by Eric Fernandez and Sylvie Marull-Tufeu at Yves Rocher (Issy les Moulineaux, France).

\section{Skin preparation}

Human skin samples were cut, embedded in Tissue Tek ${ }^{\circledR}$ and immersed into liquid nitrogen before being stored at $-20^{\circ} \mathrm{C}$. Skin sections (5 $\mathrm{mm}$ thickness) were collected on
$1 \mathrm{~mm}$ thick ZnS slides and stored at ambient temperature prior to IR analysis. The samples were kindly provided by Céline Laperdrix at Yves Rocher (Issy les Moulineaux, France).

\section{Meteorite}

A fragment of the Paris carbonaceous chondrite was crushed in a diamond compression cell to obtain a $50 \times 40 \times 3 \mu \mathrm{m}^{3}$ grain. ${ }^{15}$ The diamond compression cell was opened and the grain was measured on the $1 \mathrm{~mm}$ thick diamond window.

\section{FT-IR microspectroscopy}

Synchrotron radiation confocal FT-IR

microspectroscopy

We used a synchrotron radiation source to measure IR spectra at spatial resolutions close to the size of the microstructures in the samples and as close as possible to the projected pixel size of the FPA measurements. Spectra were recorded at the SOLEIL synchrotron facility on the SMIS beamline which exploits the edge and constant field radiations of a bending magnet. ${ }^{16}$ The synchrotron delivers $450 \mathrm{~mA}$ or $500 \mathrm{~mA}$ current in the 4/4 filling mode and $3 / 4$ hybrid mode, and operates in top-up mode for injection.

Spectra were recorded in transmission on a Nicolet Continuum XL microscope (Thermo Fisher Scientific, Villebon surYvette, France) equipped with a $50 \times 50 \mu^{2}$ liquid-nitrogen-cooled MCT/A detector (narrow-band, $650 \mathrm{~cm}^{-1}$ cut-off), a 32× NA 0.65 Schwarzschild objective and matching $32 \times$ condenser and a Prior Proscan $X Y Z$ motorised stage with minimum step size of $1 \mu \mathrm{m}$. The microscope was coupled to a Nicolet 5700 spectrometer (Thermo Fisher, Villebon sur Yvette, France) equipped with a Michelson interferometer and a $\mathrm{KBr}$ beamsplitter. The spectrometer and the microscope are constantly purged with dry air from a Parker-Balston FT-IR purge gas generator for atmospheric control. The confocal aperture was set to obtain a spatial resolution of $6 \times 6 \mu m^{2}$ for the hair sections and meteorite sample, $8 \times 8 \mu \mathrm{m}^{2}$ for the skin section to approximately match the stratum corneum thickness, and $5 \times 5 \mu \mathrm{m}^{2}$ for some selected regions of the meteorite. The microscope was operated in single-aperture mode. Spectra were recorded in transmission mode with 128 scans at $8 \mathrm{~cm}^{-1}$ resolution. Maps were recorded by raster scanning the sample with steps of $3 \mu \mathrm{m}$ in $X$ and in $Y$ directions. 


\section{FPA imaging}

Infrared spectral images were recorded at the SMIS beamline on a Cary 620 IR microscope (Agilent, Les Ulis, France) coupled to a Cary 670 spectrometer using a $\mathrm{KBr}$ beamsplitter and a Michelson interferometer. The microscope is equipped with a $128 \times 128$ pixels Stingray MCT detector. The images were measured with $4 \times / 0.2$, $15 \times / 0.61$ and $25 \times / 0.81$ (magnification/numerical aperture) objectives giving a field of view (FoV) of, respectively, $2400 \times 2400,704 \times 704$ and $422 \times 422 \mu m^{2}$ at standard magnification. The projected pixel sizes were, respectively, $20 \times 20,5.5 \times 5.5$ and $3.3 \times 3.3 \mu \mathrm{m}^{2}$ at standard magnification. The microscope is also equipped with "high-magnification" optics giving an additional magnification for a final $62.5 \times$ magnification. Images were thus also measured at $62.5 \times$ magnification with the $25 \times 0.81$ NA objective operated at high magnification with a FoV of $84 \times 84 \mu \mathrm{m}^{2}$ and projected pixel size of $0.65 \times 0.65 \mu^{2}$. Spectra were recorded in transmission mode at $8 \mathrm{~cm}^{-1}$ resolution between $850 \mathrm{~cm}^{-1}$ and $3950 \mathrm{~cm}^{-1}$ with 256 coadded scans at $2.5 \mathrm{~Hz}$ scanning speed.

\section{Data analysis}

Spectra were baseline corrected by subtracting a linear baseline in the $2800-3650 \mathrm{~cm}^{-1}$ or in the $900-1800 \mathrm{~cm}^{-1}$ range as appropriate using the Orange/Quasar ${ }^{17,18}$ and Omnic (Thermo Fisher Scientific, Villebon sur Yvette, France) software.
The integrated areas of the bands of interest were computed in Orange/Quasar and in Omnic with the parameters given in Table 1.

The contrast $C$ of a spectral image at a given wavelength was computed using the following equation:

$$
C=\frac{I_{\max }-I_{\min }}{I_{\max }+I_{\min }}
$$

where $I_{\max }$ is the maximum and $I_{\min }$ is the minimum absorption at the wavelength in the spectral image.

\section{Selection of representative spectra and computation of average spectra}

The spectra from the hair medulla and from the skin stratum corneum were selected by their higher lipid: protein ratio. The spectra located at the cortex-medulla edge and at the stratum corneum-stratum granulosum edge, exhibiting a lower lipid: protein ratio, were eliminated before computing the average spectra to keep only the most representative spectra.

\section{Results}

\section{Hair data}

The hair is an interesting sample for evaluating the accuracy, spatial resolution and contrast of FT-IR microspectroscopy measurements. Hair sometimes presents a single cell, 5-6 $\mu \mathrm{m}$ in diameter, in the hair medulla

Table 1. Parameters used to compute peak areas and peak area ratios with the peak limits and the baseline points used in the computation.

\begin{tabular}{|l|l|l|l|}
\hline Parameter & Peak(s) & Area & Baseline \\
\hline Medulla lipids & $\mathrm{C}-\mathrm{H}$ stretching & $2800-3010 \mathrm{~cm}^{-1}$ & $2800-3010 \mathrm{~cm}^{-1}$ \\
\hline Medulla aliphatic lipids & $\mathrm{C}-\mathrm{H}$ stretching of $\mathrm{CH}_{2}$ & $2900-2945 \mathrm{~cm}^{-1}$ & $2800-3010 \mathrm{~cm}^{-1}$ \\
\hline Medulla proteins & $\mathrm{N}-\mathrm{H}$ stretching & $3225-3380 \mathrm{~cm}^{-1}$ & $2800-3650 \mathrm{~cm}^{-1}$ \\
\hline Medulla lipid esters & $\mathrm{C}=\mathrm{O}$ stretching & $1725-1750 \mathrm{~cm}^{-1}$ & $1725-1750 \mathrm{~cm}^{-1}$ \\
\hline Medulla carboxylates & $\mathrm{COO}$ asym stretching & $1565-1595 \mathrm{~cm}^{-1}$ & $1565-1595 \mathrm{~cm}^{-1}$ \\
\hline Medulla carboxylates & $\mathrm{COO}$ sym stretching & $1443-1490 \mathrm{~cm}^{-1}$ & $1443-1490 \mathrm{~cm}^{-1}$ \\
\hline Oxidised disulfide bridges & $\mathrm{S}=\mathrm{O}$ sym stretching & $1010-1066 \mathrm{~cm}^{-1}$ & $1010-1066 \mathrm{~cm}^{-1}$ \\
\hline Stratum corneum lipids & $\mathrm{C}-\mathrm{H}$ stretching & $2800-3010 \mathrm{~cm}^{-1}$ & $2800-3010 \mathrm{~cm}^{-1}$ \\
\hline Stratum corneum proteins & $\mathrm{N}-\mathrm{H}$ stretching & $3225-3380 \mathrm{~cm}^{-1}$ & $2800-3650 \mathrm{~cm}^{-1}$ \\
\hline Meteorite silicates & $\mathrm{Si}-\mathrm{O}$ stretching & $900-1200 \mathrm{~cm}^{-1}$ & $1225-1750 \mathrm{~cm}^{-1}$ \\
\hline Meteorite carbonates & $\mathrm{CO}{ }^{2-}$ asymmetric stretching & $1350-1590 \mathrm{~cm}^{-1}$ & $1350-1590 \mathrm{~cm}^{-1}$ \\
\hline
\end{tabular}

*asym: asymmetrical, sym: symmetrical 
with a slightly different chemical composition from the surrounding hair cortex (Figure 1). Both the medulla and the cortex are composed of similar macromolecules ${ }^{19}$ (keratin, keratin associated proteins, fatty acids, nucleic acids...) but the hair medulla is frequently enriched in fatty lipids and carboxylates 20,21 and exhibits lower protein concentration than the cortex of the hair. We measured the medulla of a few hair sections from different donors with the confocal microscope and with the FPA imager. The FPA imager was equipped with various objectives $(4 \times, 15 \times, 25 \times, 62.5 \times)$ with different magnifications and numerical apertures $(2 \times / 0.2,7.5 \times / 0.61,12.5 \times / 0.81$, $62.5 \times / 0.81)$ giving different projected pixel sizes (respectively $20 \times 20 \mu^{2}, 5.5 \times 5.5 \mu^{2}, 3.3 \times 3.3 \mu m^{2}$, $\left.0.65 \times 0.65 \mu^{2}\right)$. The confocal microscope was coupled to a synchrotron radiation source to measure with a projected aperture size of $6 \times 6 \mathrm{\mu m}^{2}$ close to the $5.5 \times 5.5 \mu \mathrm{m}^{2}$ projected pixel size of the $15 \times$ objective on the FPA imager. The confocal microscope is routinely operated at $6 \times 6 \mathrm{\mu m}^{2}$ projected aperture size since this aperture allows obtaining a good signal-to-noise ratio across the whole spectral range of the narrowband MCT detector $\left(650-8000 \mathrm{~cm}^{-1}\right)$ and especially for proteins that absorb at $6 \mu \mathrm{m}$. The three hair sections were fully mapped or imaged, and spectral maps were generated from the area of characteristic IR absorption peaks. While it took several hours to map a whole hair section, several hair sections could be imaged in less than 20 min with the FPA imager with the $4 \times, 15 \times$ and $25 \times$ objectives $\left(2400 \times 2400 \mu^{2}, 700 \times 700 \mu m^{2}\right.$ and

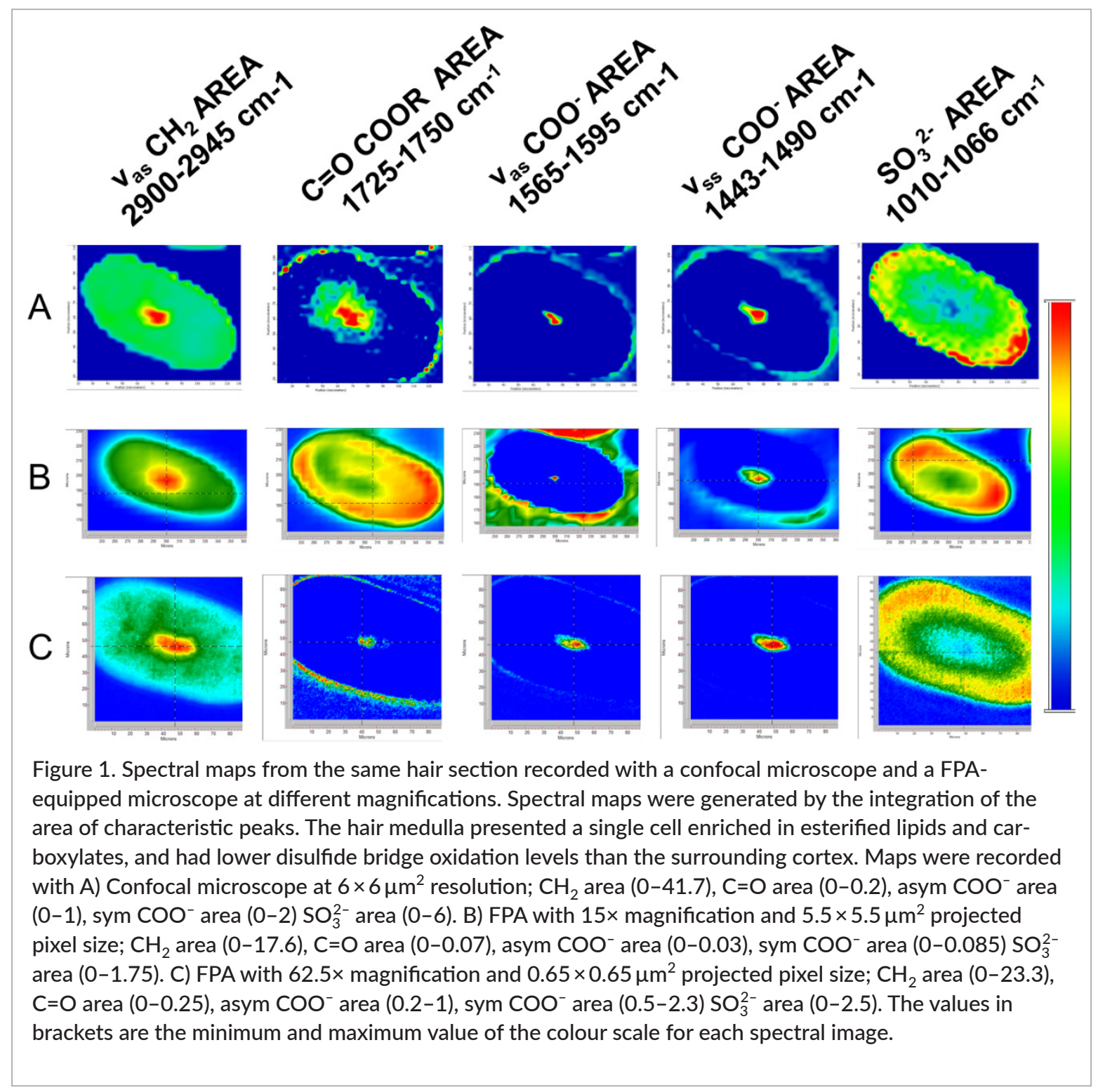


$400 \times 400 \mu m^{2}$ FoV) and a single hair section could be imaged with the $62.5 \times$ objective $\left(84 \times 84 \mu \mathrm{m}^{2} \mathrm{FoV}\right)$.

Representative spectral maps and images from hair section 1 are shown in Figure 1. The hair medulla was distinguished from the hair cortex by its lipid composition by using either the $\mathrm{C}-\mathrm{H}$ stretching peaks from lipid $\mathrm{CH}_{2}$ at $2920 \mathrm{~cm}^{-1}$, the $\mathrm{C}=\mathrm{O}$ stretching peak from esterified lipids at $1740 \mathrm{~cm}^{-1}$, the $\mathrm{C}=\mathrm{O}$ asymmetric stretching from carboxylates at $1575 \mathrm{~cm}^{-1}$ and the $S=O$ peak from oxidised disulfide bridges at $1040 \mathrm{~cm}^{-1}$. The increased lipid concentration in the medulla could be detected in the confocal map, and in the $15 \times, 25 \times$ and $62.5 \times \mathrm{FPA}$ images. The medulla could not be detected in the $4 \times$ FPA image. Yet, the hair medulla appeared smaller in the confocal microscope map shown in Figure 1A compared to the $62.5 \times$ and $15 \times$ FPA images shown in Figure $1 \mathrm{~B}$ and $1 \mathrm{C}$. In the bright field micrograph (not shown) the medulla, which could be spotted visually thanks to the darker colour due to its melanin content, represented only $4 \%$ of the hair surface. Using the $\mathrm{C}-\mathrm{H}$ stretching peak giving the lowest diffraction, the medulla surface was evaluated at $4.2 \%$ of the total hair surface in the confocal spectral map, $27 \%$ and $7.4 \%$ in the $15 \times$ and $62.5 \times$ FPA images, respectively. Another way to measure the accuracy of the spectral maps and images is to look at the contrast of the spectral images. We used the absorption intensity at the $\mathrm{CH}_{2}$ peak at $2921 \mathrm{~cm}^{-1}$ to compute the contrast. The confocal measurement generally gave the highest contrast between the medulla and the cortex followed by the $62.5 \times$ FPA image (Table 3) except for hair section 3. Note that the contrast appeared higher in the spectral images for the $\mathrm{COO}^{-}$peaks at $1575 \mathrm{~cm}^{-1}$ and
$1480 \mathrm{~cm}^{-1}$ of Figure 1 than for the $\mathrm{CH}_{2} / \mathrm{NH}$ ratio, since no carboxylate is detectable in the cortex.

We compared spectra of the medulla from the same hair section in order to see whether the concentration of the different hair constituents was accurately measured in all configurations. The average spectra from the medulla from each of the three hair sections are presented in Figure 2, showing that the confocal microscope gives a higher lipid/protein ratio due to measuring a higher lipid signal and a lower protein signal. The lipid/protein ratio evaluated by the $\mathrm{CH}_{2} / \mathrm{NH}$ peak intensity ratio is shown as boxplots in Figure 2. The lipid/protein ratios evaluated by the peak area ratios of the $\mathrm{C}-\mathrm{H}$ stretching $/ \mathrm{N}-\mathrm{H}$ stretching are given for the three hair sections in Table 2. We can see that the peak area ratio measured using a confocal microscope operated with a projected aperture size of $6 \times 6 \mu^{2}$ is $2-4$ times higher than that measured with the FPA with a comparable projected pixel size of $5.5 \times 5.5 \mu \mathrm{m}^{2}$. The $62.5 \times$ FPA image allows a closer estimation of the lipid/protein ratio but confocal measurements were still 1.05-1.87 times higher.

\section{Skin sample}

We used a human skin section deposited on a ZnS slide to evaluate the effect of diffraction for a sample composed of multiple layers. We compared a spectral map from a confocal microscope coupled to the synchrotron radiation source operated at $8 \times 8 \mu^{2}$ aperture size and a spectral image obtained with the FPA-equipped microscope with a $15 \times / 0.65 \mathrm{NA}$ objective $\left(5.5 \times 5.5 \mu^{2}\right.$ projected pixel size). Figure 3 shows spectral maps and images generated from different absorption peaks that allow the

Table 2. Values of the hair medulla $\mathrm{CH}_{2} / \mathrm{NH}$ area ratio for three hair sections measured by an aperture-less imaging microscope at different magnifications and by a confocal microscope.

\begin{tabular}{|l|c|c|c|}
\hline Mode & Hair 1 & Hair 2 & Hair 3 \\
\hline $32 \times$ confocal & $0.73 \pm 0.04$ & $0.85 \pm 0.06$ & $0.77 \pm 0.07$ \\
\hline $62.5 \times$ FPA & $0.39 \pm 0.04$ & $0.70 \pm 0.06$ & $0.74 \pm 0.06$ \\
\hline $25 \times$ FPA & $0.38 \pm 0.02$ & $0.33 \pm 0.05$ & $0.31 \pm 0.04$ \\
\hline $15 \times$ FPA & $0.35 \pm 0.03$ & $0.20 \pm 0.02$ & $0.20 \pm 0.03$ \\
\hline
\end{tabular}

Table 3. Image contrast for the lipid $\mathrm{CH}_{2}$ at $2921 \mathrm{~cm}^{-1}$.

\begin{tabular}{|l|c|c|c|}
\hline & Hair 1 & Hair 2 & Hair 3 \\
\hline Confocal & 0.76 & 0.78 & 0.60 \\
\hline $62.5 \times$ FPA & 0.47 & 0.67 & 0.67 \\
\hline
\end{tabular}



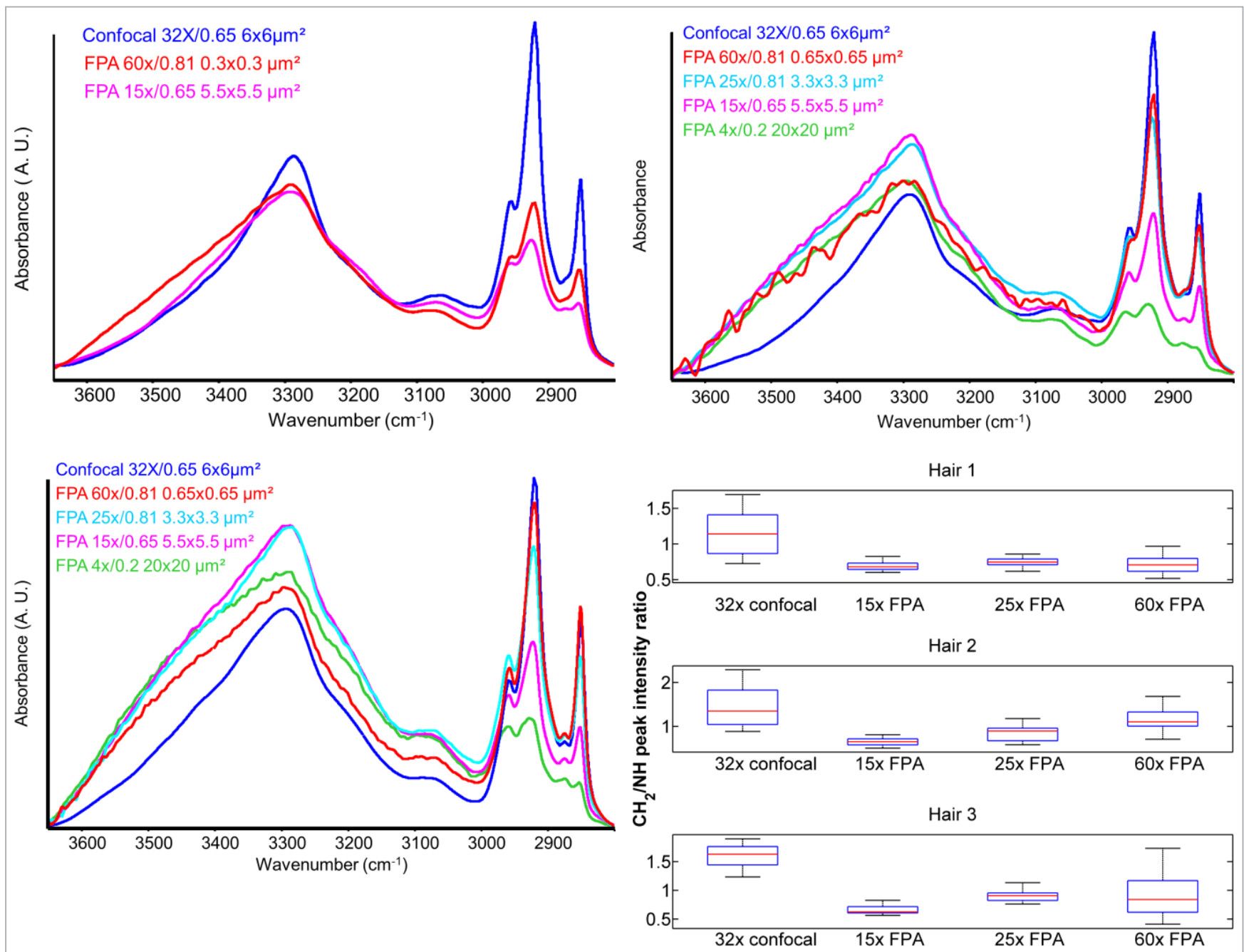

Figure 2. Comparison of spectra from the medulla of hair sections recorded with a confocal microscope and a FPA microscope at different projected pixel sizes in the $\mathrm{C}-\mathrm{H}$ and $\mathrm{N}-\mathrm{H}$ stretching region $\left(2800-3650 \mathrm{~cm}^{-1}\right)$. A) hair section 1 , B) hair section 2, C) hair section 3. Figure colours: blue: confocal SR- $\mu$ FTIR, red 62.5× FPA, turquoise $25 \times$ FPA, magenta $15 \times$ FPA, green $4 \times$ FPA. The $\mathrm{CH} / \mathrm{NH}$ ratio appears lower in the FPA spectra compared to the confocal spectra. The $\mathrm{C}-\mathrm{H}$ stretching peaks seem lower and the $\mathrm{N}-\mathrm{H}$ peak seems higher in the FPA data. D) Box plots showing the distribution of the $\mathrm{CH}_{2} / \mathrm{NH}$ peak intensity ratio for the three hair sections and the various magnifications. Red line: median, blue box first and third quartiles, whiskers: extreme values.

different skin compartments to be differentiated. The uppermost layer, the stratum corneum (SC) made of dead keratinocytes having lost their nucleus and organelles (corneocytes), can be evidenced by its increased lipid content. The underneath layer, the stratum granulosum made of living cells at different stages of differentiation toward the corneocyte stage, is evidenced by its amide III peak. The underlying dermis can be distinguished by its collagen content. All compartments can be detected and characterised with confocal maps and FPA images.

We use the SC spectra to evaluate whether diffraction affects the spectral signature of skin since SC is the thin- nest layer of the epidermis, being only 8-10 $\mathrm{mm}$ thick. $\mathrm{SC}$ exhibits strong characteristic $\mathrm{C}-\mathrm{H}$ peaks between $2800 \mathrm{~cm}^{-1}$ and $3000 \mathrm{~cm}^{-1}$ and a $\mathrm{N}-\mathrm{H}$ peak at $3285 \mathrm{~cm}^{-1}$. As for hair medulla, the increased lipid/protein ratio relative to the surrounding tissue can be used to identify the SC, evaluate the accuracy of the measurement and the effect of diffraction. The $\mathrm{CH} / \mathrm{NH}$ peak area ratio was found to be $0.27 \pm 0.03$ in the confocal microscope map and $0.13 \pm 0.06$ in the $15 \times$ FPA data, a two times difference. It should also be noted that the intensity of the SC spectra is globally inferior for the FPA than for the confocal measurement. For example, the average 


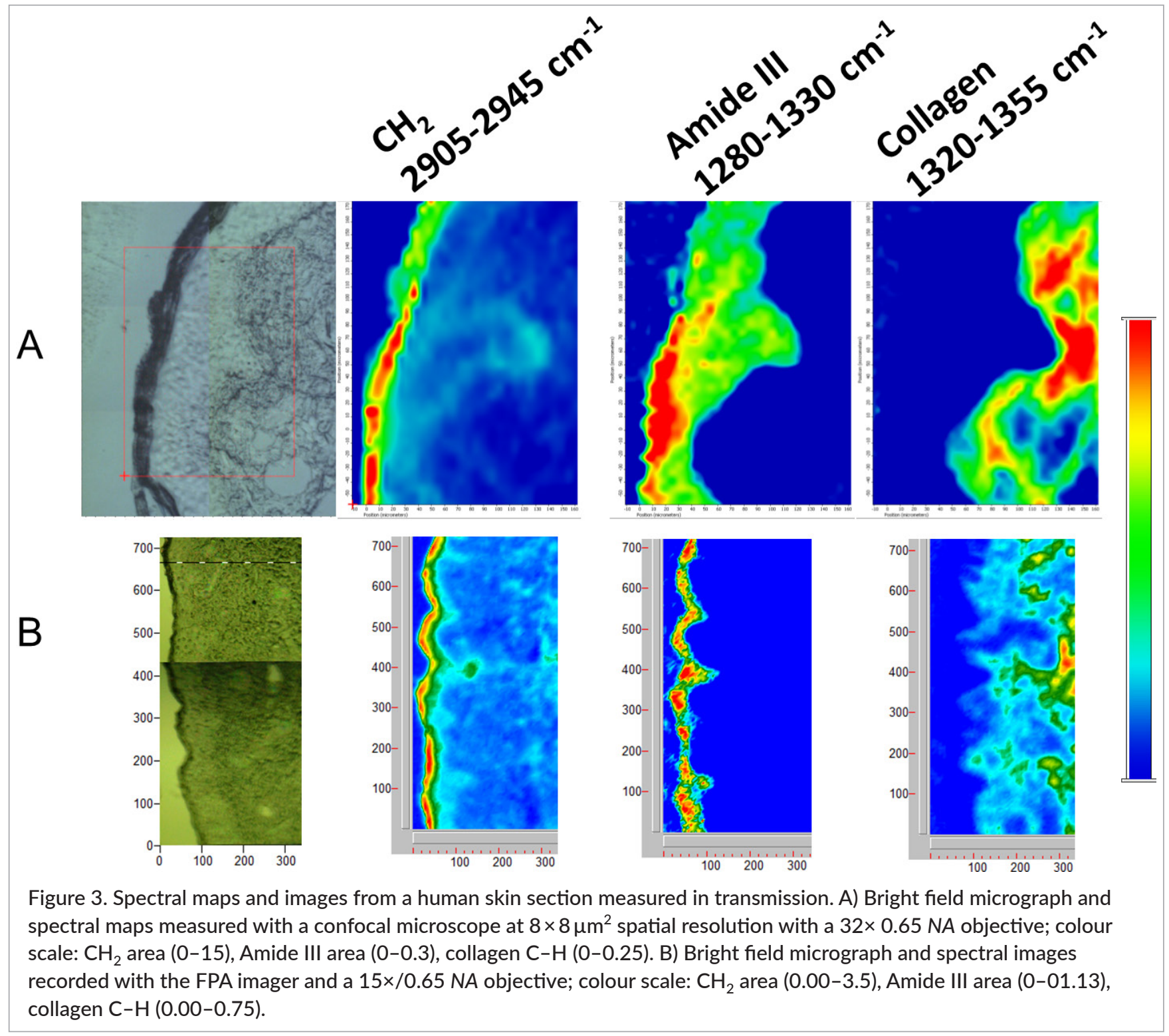

intensity of the $\mathrm{N}-\mathrm{H}$ peak at $3285 \mathrm{~cm}^{-1}$ for the $\mathrm{SC}$ is 0.54 in the confocal measurement, while it is only 0.32 in for the 15× FPA image. The SC spectra from the FPA also present a wider $\mathrm{NH}$ peak with a stronger shoulder at $3450 \mathrm{~cm}^{-1}$ suggesting the presence of $\mathrm{O}-\mathrm{H}$ absorption. This shoulder is not seen in the confocal spectra.

\section{Meteorite sample}

Unlike biological samples used in this study, the meteorite sample exhibits a fine grain structure with radically different chemical compositions. The meteorite matrix is composed of amorphous silicate grains and contains inclusions from other silicates (olivine, serpentine, diopside) as well as sulfate and calcium carbonate inclusions, and organic carbonaceous inclusions. ${ }^{15}$ These various components can be mapped by the area of characteristic peaks (Figure 5): silicates via the $\mathrm{Si}-\mathrm{O}$ stretching peak between $900 \mathrm{~cm}^{-1}$ and $1200 \mathrm{~cm}^{-1}$, organic phases by the $\mathrm{C}-\mathrm{H}$ stretching peaks between $2800 \mathrm{~cm}^{-1}$ and $3000 \mathrm{~cm}^{-1}$ and the $\mathrm{C}-\mathrm{C} / \mathrm{C}-\mathrm{N}$ stretching peak between $1500 \mathrm{~cm}^{-1}$ and $1715 \mathrm{~cm}^{-1}$, water trapped in amorphous silicates between $3000 \mathrm{~cm}^{-1}$ and $3650 \mathrm{~cm}^{-1}$, and mineral $\mathrm{O}-\mathrm{H}$ by the structural $\mathrm{O}-\mathrm{H}$ peak between $3600 \mathrm{~cm}^{-1}$ and $3700 \mathrm{~cm}^{-1}$. Carbonates give a characteristic $\mathrm{V}_{3} \mathrm{CO}_{3}^{2-}$ antisymmetric stretching peak at around $1450 \mathrm{~cm}^{-1}$. The Paris meteorite fragment used in this study contains two small carbonate particles, particle 1 located inside the fragment and particle 2 located at the edge. This allows us to evaluate the relative performances of the confocal and aperture-less microscopes for smaller 


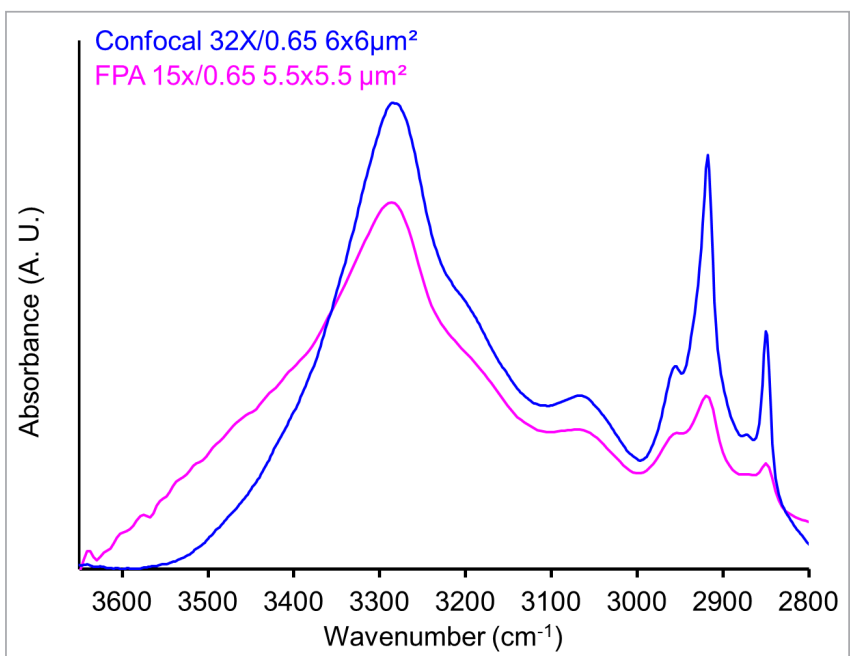

Figure 4. Average spectra of the stratum corneum from the skin section shown in Figure 3. The spectra were recorded with a confocal microscope (blue) and a FPA imager (magenta) at $15 \times$. The blue spectrum shows higher absorption and 2.7 times higher $\mathrm{CH} / \mathrm{NH}$ ratio. The $\mathrm{CH} / \mathrm{NH}$ ratio is inversed in the two spectra. The $\mathrm{NH}$ peak width appears larger in the magenta spectrum and presents a shoulder at $3450 \mathrm{~cm}^{-1}$ suggesting the presence of an $\mathrm{O}-\mathrm{H}$ stretching peak not seen in the blue spectrum.

particles absorbing at longer wavelengths than the $\mathrm{C}-\mathrm{H}$ and $\mathrm{N}-\mathrm{H}$ in the hair samples. The confocal map and FPA image in Figure 5 show different carbonate particle sizes and the spectra of the particles shown in Figure 6 different evaluations of the carbonate content. Particle 1 located inside the fragment appears slightly smaller than particle 2 in the FPA image, but larger in the confocal map. Spectra of the carbonate particles in Figure 6 show different silicate/carbonate ratios. Particle 1 located inside the meteorite fragment has a carbonate/silicate ratio of 0.15 as measured by the confocal microscope and 0.14 by the imaging microscope. Particle 2 located at the edge of the fragment had a higher carbonate/silicate ratio than particle 1: 0.77 as measured by the imaging microscope but only 0.38 by the confocal microscope. However, particle 2 was smaller than particle 1 when measured by Raman microscopy. This suggested that the IR microscopes both overestimated the size and underestimated the carbonate/silicate ratio of particle 2 located at the edge, particularly in the FPA images. It can also be noticed that diffraction rings are visible around particle 1 and 2 in the FPA image due to their strong contrast with the surrounding grains. This also shows that the carbonate particles give spurious signal at empty pixels outside of the meteorite fragment.

\section{Overall spectral intensity}

We observed that the spectra measured with the FPA imaging microscope were less intense than the spectra from the same sample measured with the confocal instrument, especially for low magnification objectives. In Figure 7A, we can see spectra from the cortex of the same hair section measured with different objectives $(4 \times, 15 \times, 25 \times, 62.5 \times)$ with the FPA imaging microscope and with the confocal microscope at $32 \times$ magnification. The confocal measurement produces the most intense spectrum in the amide $\left(1700-1480 \mathrm{~cm}^{-1}\right)$ and in the fingerprint region $\left(1480-900 \mathrm{~cm}^{-1}\right)$, while the $4 \times$ produces a spectrum that is 2-2.5 times less intense (for amide II and amide I, respectively). Even at $15 \times$ magnification, the intensity of the amide peaks is 1.3 and 1.5 times less intense than for the confocal measurement (for amide II and amide I, respectively). The $62.5 \times$ and $25 \times$ magnifications give similar intensity for the cortex, but slightly underestimate the intensity of the signal compared to the confocal measurement by 1.2-1.3 times. This effect was less marked at shorter wavelengths and the $25 \times$ and $62.5 \times$ objectives gave almost exactly the same estimation of the peak intensity for the $\mathrm{N}-\mathrm{H}$ peak as the confocal measurement in that sample but differences could be observed for other samples (Figure 2A). In order to quantify the effect of the objective magnification on spectra intensity we plotted the intensity of one peak versus the relative number of sample-free pixels in the image (pixels without contribution from the sample). The $\mathrm{N}-\mathrm{H}$ peak was chosen as a reporter for spectral intensity and its area was computed for the cortex of all three sections at every magnification (after excluding the hair medulla spectra with a K-means clustering in Orange/ Quasar). In Figure 7B we can see the relationship between magnification and spectral intensity. Each section had a different thickness but, for all sections, the $25 \times$ and $62.5 \times$ magnification objectives gave higher absorption values than the $4 \times$ and $15 \times$. The $32 \times$ confocal system generally gave more intense peaks.

\section{Discussion}

\section{Spectral and spatial accuracy}

We measured spectral maps and images of the same representative, real-life, microstructured samples with a confocal IR microscope coupled to a synchrotron source 


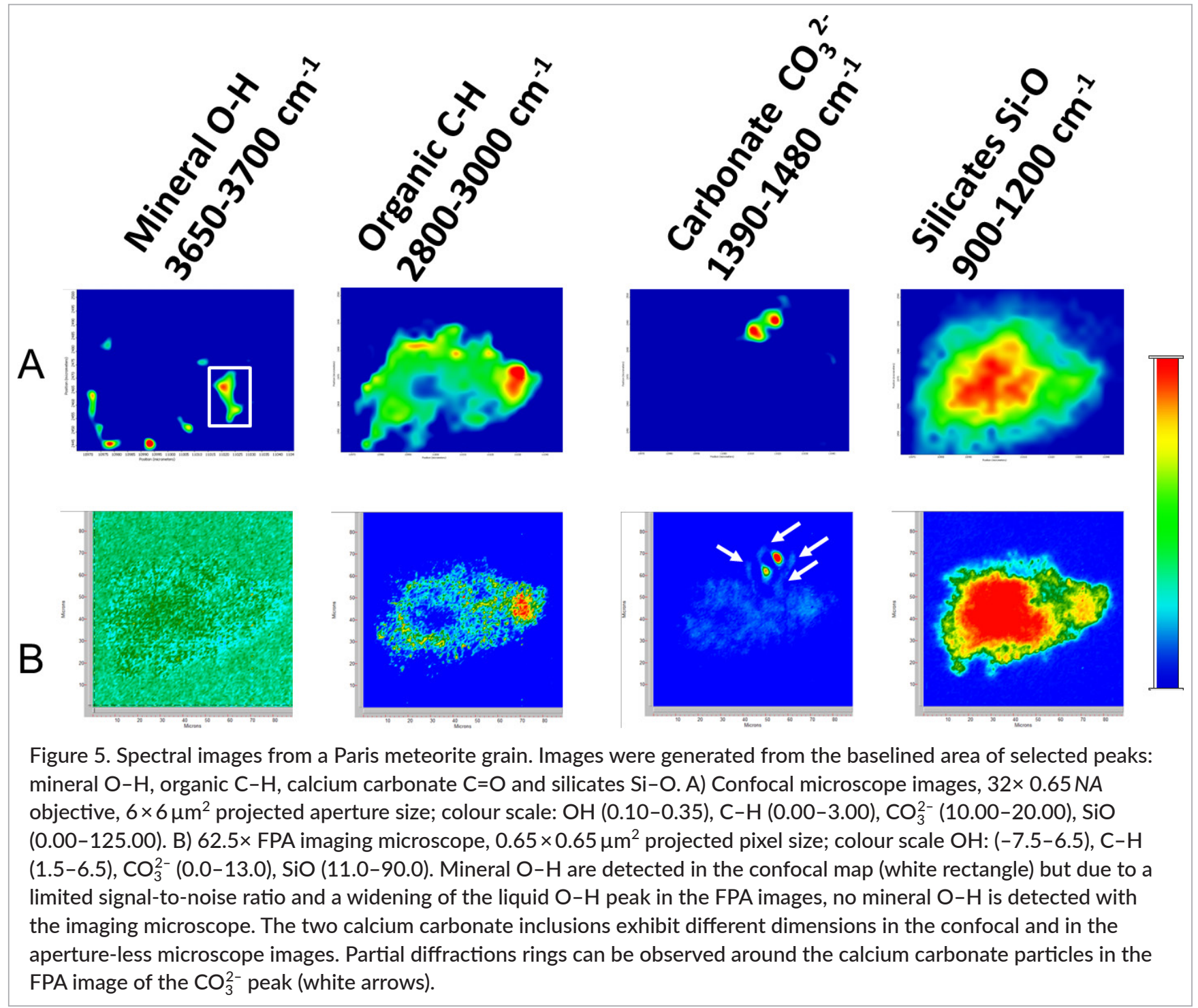

and with an aperture-less infrared imaging microscope. We observed that the confocal microscope and the imaging microscope gave different spectra for the same microscopic feature. As the confocal configuration is used in IR microspectroscopy to reduce the contribution of diffraction in the spectra and therefore improve the lateral resolution, ${ }^{1}$ it represented our gold standard for spatial resolution and spectral accuracy. The apertureless imaging system showed a different composition of the micron-size features. The size of the features was overestimated in both systems (as seen by comparing the sizes in the IR maps and bright field micrographs), but more so in the aperture-less spectral images. The accuracy of the aperture-less images strongly depended on the magnification and numerical aperture of the optical system when comparing different objectives.
For the single lipid-rich cell in the hair medulla, we observed that the lipid/protein ratio was always the highest in the confocal measurements compared to the different aperture-less spectral images and that the area of the medulla was also better estimated in the confocal measurements. The medulla could not be detected in the $4 \times$ magnification FPA images. In the $15 \times$ magnification FPA images, the lipid/protein ratio was underestimated by a factor of 2 to 4 depending on the sample. This strong variability could be due to the geometry of the hair medulla. Hair section 1 medulla was narrow and elongated (short axis of 5-6 $\mu \mathrm{m}$ and long axis of $25-30 \mu \mathrm{m}$ ) while the medulla of hair 3 was more round and symmetrical ( $12 \mu \mathrm{m}$ diameter) and gave the most accurate estimate. The accuracy of the measurement of a particle will thus strongly depend on 


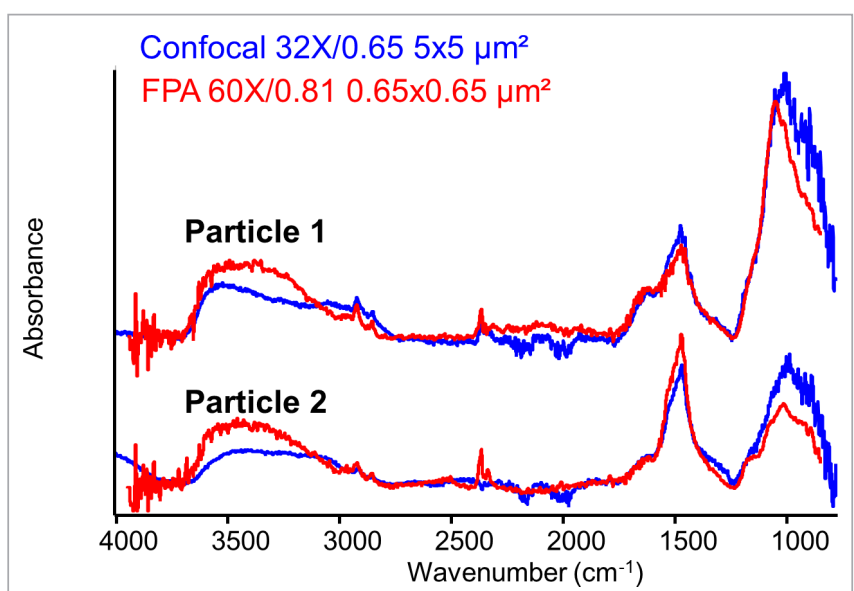

Figure 6. Spectra of carbonate particles 1 and 2 in the Paris meteorite fragment recorded by the confocal and the imaging microscope. Particle 1 is located inside the fragment and particle 2 is located at the edge. The spectra from the imaging microscope are the average of five to six spectra from each particle and the confocal spectra are individual spectra. Spectra were offset for clarity.

the shape, size and aspect ratio of the particle. If one dimension is smaller than some threshold (depending on sample and microscope optical properties) it would limit the accuracy of the measurement. It could thus be difficult to predict when aperture-less FPA measurements would be accurate. The best results from the aperture-less system were obtained in the $62.5 \times$ magnification FPA images. The lipid/protein ratio estimation in hair section 3 differed by only a few percent from the confocal measurement. However, for hair section 1, the lipid/protein ratio was different by a factor of nearly two from the confocal measurement. This was both due to an underestimation of the lipid content and to an overestimation of the protein content in the medulla. The confocal system was able to accurately detect both the lipid increase and the decrease in the protein content of the medulla.

In the skin SC, the lipid/protein ratio in the $15 \times$ magnification FPA image was underestimated by a factor of nearly two compared to the confocal measurement. Even if the SC width was 16-20 $\mathrm{m}$, 5-6 times larger than the wavelength used to probe it, and several hundred microns in length. Diffraction from the surrounding empty area and neighbouring cell layers (poor in lipids) may have been responsible for the underestimation of the lipid concentration and overestimation of the protein concentration. Thus, even for layered samples that have

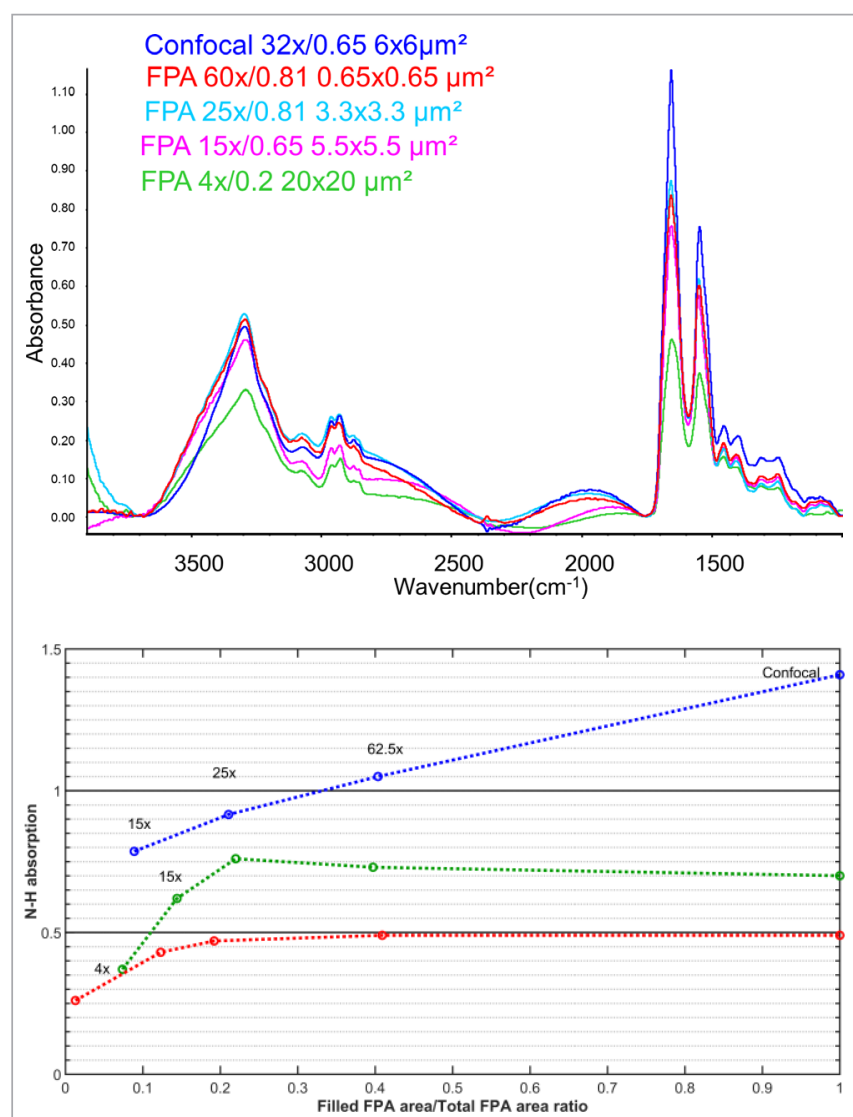

Figure 7. Comparison of the overall intensity of the spectra of one hair section recorded at different magnifications with the aperture-less FPA imaging microscope and the confocal microscope. Top) Average spectra of 4-16 representative spectra from the cortex of hair section 2 at $4 \times, 15 \times, 25 \times$ and $62.5 \times$ magnifications with the FPA imager and of 20 spectra from the cortex at $32 \times$ magnification with the confocal system. Spectra were baseline corrected to remove shifts in the absorbance axis but not normalised as to conserve intensity. Bottom) Intensity of the hair spectra (area of the NH peak for the cortex) as a function of the full/empty area ratio in the FPA image. The confocal measurement was assumed to have a ratio of 1 .

one dimension much greater than the diffraction limit, the aperture-less system may misrepresent the chemical composition of the layers.

For all biological samples we used the $\mathrm{N}-\mathrm{H}$ and $\mathrm{C}-\mathrm{H}$ signals that are found at $3290 \mathrm{~cm}^{-1}$ and $2800-3010 \mathrm{~cm}^{-1}$, respectively ( $3 \mu \mathrm{m}$ and $3.5-3.3 \mu \mathrm{m}$ wavelengths), to compute the lipid/protein ratio and compare the accuracy of the confocal and aperture-less microscopes. These are the shortest useful wavelengths in the mid-IR, for which diffraction is the lowest. We can expect that the inaccuracies will be stronger at longer wavelengths. 
In the meteorite fragment, we used the calcium carbonate signal found at longer wavelength $\left(1470 \mathrm{~cm}^{-1}\right.$ or $6.8 \mu \mathrm{m})$ to estimate the accuracy of the FPA and confocal measurements. Both systems overestimated the size of the calcium carbonate particles (their actual size was found by Raman microscopy at $0.5 \mu \mathrm{m}$ spatial resolution) but more so for the aperture-less microscope. It could be seen that the error was also dependent on the location of the particle. This was especially seen for particle 2 located at the edge of the fragment. The contribution from the silicate matrix appeared lower in the FPA image, probably due to the empty area surrounding the particle. This particle also created diffraction rings that were clearly visible in the empty pixels outside the sample, and thus also contributed to add some carbonate signal to the other pixels inside the grain.

The reported changes in peak ratios could have an influence in quantitative and qualitative analysis. For example, it could influence quantitative models by multivariate regression in complex spatially heterogeneous samples when using FPA microscopes.

It could also have an impact in cluster analyses by decreasing the discriminant power of the spectra of neighbouring parts of the sample. For example, when trying to develop automatic classification models in spectral histopathology to separate closely resembling spectra from adjacent cell layers; or to detect rare pathological cells among thousands of healthy cells in a smear.

\section{Spectral intensity}

We observed that the hair section spectra showed lower absorbance when measured in the $4 \times$ and $15 x$ FPA images compared to the $32 \times$ confocal, $25 \times$ and $62.5 \times$ FPA measurements. This was also observed for the skin section at $15 \times$ magnification. The intensity of the peaks seemed to decrease with lower magnification of the objective in the FPA system and was highest with the confocal microscope although it used a lower magnification. This may be similar to what happens in single-point measurements when measuring an isolated particle with a projected aperture size much larger than the particle itself. Part of the light does not interact with the particle and therefore apparent absorption appears lower. This suggests that every pixel of the FPA sees a part of the image that is sample-free. In other words, it means that every part of the detector FoV, even sample-free parts, contributes to the signal read by every pixel of the FPA. This can be caused by scattering and diffraction within the sample and optics, and could be understood in terms of wavefront propagation for the whole optical system. Since peak intensity is often used to compute concentrations using the Beer-Lambert-Bouguer law, it could be important to be aware of the effect of magnification when using aperture-less systems.

\section{Conclusion}

Since fast IR imaging is crucial for studying large samples, especially for biomedical applications, several groups have reported modifying IR imagers for restoring high spatial resolution by physical or computational means. ${ }^{22-25}$ However, the performance was always evaluated on image fidelity and contrast at one wavelength and overall spectral accuracy was often not reported. Image deconvolution (FSD-deconvolution, PSF-deconvolution) techniques ${ }^{26-28}$ were proposed to enhance image resolution, and the use of higher magnification (74x) and/or higher numerical aperture objectives was advocated to restore image resolution. We confirm here that higher magnification objectives can give more accurate results, but do not always achieve the best accuracy. Image restoration results are often judged in terms of image improvement rather than in terms of spectral accuracy. Image deconvolution methods and optical configuration optimisation were shown to improve the definition of the spectral images and it would be interesting to know if they can improve the spectral accuracy. Here we compared spectral accuracy from a confocal microscope and an aperture-less imaging microscope on the same position from the same samples and showed that even at higher magnification and NA, the spectra from the imaging system could differ significantly from the confocal microscope gold standard. This could have crucial implications, especially for biomedical applications where different samples often exhibit minor spectral differences. For example in order to diagnose the presence of cells resistant to anticancerous agents in chronic myeloid leukemia ${ }^{29}$ it may be necessary to find a unique diseased cell in the middle of hundreds of thousands of healthy cells.

\section{References}

1. G.L. Carr, "Resolution limits for infrared microspectroscopy explored with synchrotron radia- 
tion", Rev. Sci. Instrum. 72, 1613 (2001). https://doi. org/10.1063/1.1347965

2. F. Jamme, B. Lagarde, A. Giuliani, G.A. Garcia and L. Mercury, "Synchrotron infrared confocal microscope: application to infrared 3D spectral imaging", J. Phys. Conf. Ser. 425(14), 142002 (2013). https://doi. org/10.1088/1742-6596/425/14/142002

3. J. Jonkman and C.M. Brown, "Any way you slice it-a comparison of confocal microscopy techniques", J. Biomol. Tech. 26(2), 54 (2015). https://doi. org/10.7171/jbt.15-2602-003

4. M.J. Hackett, S. Caine, X. Liu, T.E. May and F. Borondics, "Development of single-beam widefield infrared imaging to study sub-cellular neuron biochemistry", Vib. Spectrosc. 77, 51 (2015). https:// doi.org/10.1016/j.vibspec.2014.12.004

5. B.R. Wood, M. Kiupel and D. McNaughton, "Progress in Fourier transform infrared spectroscopic imaging applied to venereal cancer diagnosis", Vet. Pathol. 51(1), 224 (2014). https://doi. org/10.1177/0300985813501340

6. J. Nallala, M.-D. Diebold, C. Gobinet, O. Bouché, G.D. Sockalingum, O. Piot and M. Manfait, "Infrared spectral histopathology for cancer diagnosis: a novel approach for automated pattern recognition of colon adenocarcinoma", Analyst 139(16), 4005 (2014). https://doi.org/10.1039/C3AN01022H

7. E. Levenson, P. Lerch and M.C. Martin, "Spatial resolution limits for synchrotron-based spectromicroscopy in the mid- and near-infrared", J. Synchrotron Radiat. 15(Pt 4), 323 (2008). https://doi.org/10.1107/ S0909049508004524

8. P. Lasch and D. Naumann, "Spatial resolution in infrared microspectroscopic imaging of tissues", Biochim. Biophys. Acta - Biomembr. 1758(7), 814 (2006). https://doi.org/10.1016/j. bbamem.2006.06.008

9. M.J. Nasse, E.C. Mattson, R. Reininger, T. Kubala, S. Janowski, Z. El-Bayyari and C.J. Hirschmugl, "Multibeam synchrotron infrared chemical imaging with high spatial resolution: beamline realization and first reports on image restoration", Nucl. Instruments Methods Phys. Res. Sect. A Accel. Spectrometers, Detect. Assoc. Equip. 649(1), 172 (2011). https://doi. org/10.1016/j.nima.2010.12.095

10. E. Levenson, P. Lerch and M.C. Martin, "Infrared imaging: synchrotrons vs. arrays, resolution vs. speed", Infrared Phys. Technol. 49(1-2), 45 (2006). https://doi.org/10.1016/j.infrared.2006.01.026

11. E. Levenson, P. Lerch and M.C. Martin, "Spatial resolution limits for synchrotron-based spectromicroscopy in the mid- and near-infrared", J. Synchrotron Radiat. 15(4), 323 (2008). https://doi.org/10.1107/ S0909049508004524

12. K. Yeh, S. Kenkel, J.-N. Liu and R. Bhargava, "Fast infrared chemical imaging with a quantum cascade laser", Anal. Chem. 87(1), 485 (2015). https://doi. org/10.1021/ac5027513

13. J.A. Kimber and S.G. Kazarian, "Spectroscopic imaging of biomaterials and biological systems with FTIR microscopy or with quantum cascade lasers", Anal. Bioanal. Chem. 409(25), 5813 (2013). https://doi. org/10.1007/s00216-017-0574-5

14. Stelzer, "Contrast, resolution, pixelation, dynamic range and signal-to-noise ratio: fundamental limits to resolution in fluorescence light microscopy", J. Microsc. 189(1), 15 (1998). https://doi.org/10.1046/ j.1365-2818.1998.00290.x

15. Z. Dionnet, A. Aleon-Toppani, D. Baklouti, F. Borondics, F. Brisset, Z. Djouadi, C. Sandt and R. Brunetto, "Organic and mineralogic heterogeneity of the Paris meteorite followed by FTIR hyperspectral imaging", Meteorit. Planet. Sci. 53(12), 2608 (2018). https://doi.org/10.1111/maps.13178

16. P. Dumas, F. Polack, B. Lagarde, O. Chubar, J.L. Giorgetta and S. Lefrançois, "Synchrotron infrared microscopy at the French synchrotron facility Soleil", Infrared Phys. Technol. 49(1-2), 152 (2006). https://doi.org/10.1016/j.infrared.2006.01.030

17. M. Toplak, G. Birarda, S. Read, C. Sandt, S.M. Rosendahl, L. Vaccari, J. Demšar and F. Borondics, "Infrared Orange: connecting hyperspectral data with machine learning", Synchrotron Radiat. News 30(4), 40 (2017). https://doi.org/10.1080/089 40886.2017.1338424

18. J. Demšar, T. Curk, A. Erjavec, T. Hočevar, M. Milutinovič, M. Možina, M. Polajnar, M. Toplak, A. Starič, M. Stajdohar, L. Umek, L. Zagar, J. Zbontar, M. Zitnik and B. Zupan, "Orange: data mining toolbox in Python", J. Mach. Learn. Res. 14, 2349 (2013).

19. P.M.J. Barton, A Forensic Investigation of Single Human Hair Fibres Using FTIR-ATR Spectroscopy and Chemometrics. Thesis, Queensland University 
of Technology (2011). https://eprints.qut.edu. au/41873/1/Paul_Barton_Thesis.pdf

20. L. Kreplak, F. Briki, Y. Duvault, J. Doucet, C. Merigoux, F. Leroy, J.L. Lévêque, L. Miller, G.L. Carr, G.P. Williams and P. Dumas, "Profiling lipids across Caucasian and Afro-American hair transverse cuts, using synchrotron infrared microspectrometry", Int. J. Cosmet. Sci. 23(6), 369 (2001). https://doi.org/10.1046/j.0412-5463.2001.00118.x

21. F. Briki, B. Busson, L. Kreplak, P. Dumas and J. Doucet, "Exploring a biological tissue from atomic to macroscopic scale using synchrotron radiation: example of hair.", Cell. Mol. Biol. (Noisy-le-grand). 46(5), 1005 (2000).

22. D. Mayerich, T. van Dijk, M.J. Walsh, M.V. Schulmerich, P.S. Carney and R. Bhargava, "On the importance of image formation optics in the design of infrared spectroscopic imaging systems", Analyst 139(16), 4031 (2014). https://doi. org/10.1039/c3an01687k

23. R.K. Reddy, M.J. Walsh, M.V. Schulmerich, P.S. Carney and R. Bhargava, "High-definition infrared spectroscopic imaging", Appl. Spectrosc. 67(1), 93 (2013). https://doi.org/10.1366/11-06568

24. M.J. Nasse, M.J. Walsh, E.C. Mattson, R. Reininger, A. Kajdacsy-Balla, V. Macias, R. Bhargava and C.J. Hirschmugl, "High-resolution Fourier-transform infrared chemical imaging with multiple synchrotron beams", Nat. Methods 8(5), 413 (2011). https://doi. org/10.1038/nmeth.1585
25. E.C. Mattson, M.J. Nasse, M. Rak, K.M. Gough and C.J. Hirschmugl, "Restoration and spectral recovery of mid-infrared chemical images", Anal. Chem. 84(14), 6173 (2012). https://doi.org/10.1021/ac301080h

26. P. Lasch, A. Pacifico and M. Diem, "Spatially resolved IR microspectroscopy of single cells", Biopolymers 67(4-5), 335 (2002). https://doi.org/10.1002/ bip.10095

27. I. Amenabar, S. Poly, M. Goikoetxea, W. Nuansing, P. Lasch and R. Hillenbrand, "Hyperspectral infrared nanoimaging of organic samples based on Fourier transform infrared nanospectroscopy", Nat. Commun. 8, 14402 (2017). https://doi.org/10.1038/ ncomms 14402

28. E.C. Mattson, M. Unger, S. Clède, F. Lambert, C. Policar, A. Imtiaz, R. D'Souza and C.J. Hirschmugl, "Toward optimal spatial and spectral quality in widefield infrared spectromicroscopy of IR labelled single cells.", Analyst 138(19), 5610 (2013). https://doi. org/10.1039/c3an00383c

29. C. Sandt, O. Feraud, M.-L. Bonnet, C. Desterke, R. Khedhir, S. Flamant, C.G. Bailey, J.E.J. Rasko, P. Dumas, A. Bennaceur-Griscelli and A.G. Turhan, "Direct and rapid identification of T315i-mutated BCR-ABL expressing leukemic cells using infrared microspectroscopy", Biochem. Biophys. Res. Commun. 503(3), 1861 (2018). https://doi.org/10.1016/j. bbrc.2018.07.127 